Pacific Journal of Mathematics

CROSSED PRODUCTS AND GALOIS EXTENSIONS OF HOFF

BERT JAMES BLATTNER AND SUSAN MONTGOMERY 


\title{
CROSSED PRODUCTS AND GALOIS EXTENSIONS OF HOPF ALGEBRAS
}

\author{
Robert J. Blattner and Susan Montgomery \\ To the memory of our friend Henry A. Dye
}

\begin{abstract}
In this paper we explore further the subject of crossed products $A \#_{\sigma} H$ of an arbitrary Hopf algebra $H$ (weakly) acting on a noncommutative algebra $A$ over a field $k$. These general crossed products, which play a fundamental role in the theory of extensions of Hopf algebras, were introduced independently by Y. Doi and M. Takeuchi and by the present authors and $M$. Cohen. Here we give several characterizations of crossed products $A \#_{\sigma} H$ with invertible cocycle $\sigma$. These characterizations are then used to extend to such crossed products known results for smash products concerning duality and Maschke-type theorems. We also prove a Noether-Skolem theorem using these methods.
\end{abstract}

Our first main result is that a crossed product $B=A \#_{\sigma} H$ with $\sigma$ invertible is a cleft $H$-comodule algebra. Doi and Takeuchi had proved earlier that cleft comodule algebras are crossed products with $\sigma$ invertible [DT], and thus this property characterizes such crossed products. We note that a weaker version of the [DT] result was proved in [BCM], with essentially the same proof.

Combining this characterization with a second result in [DT], it follows that $B \cong A \#_{\sigma} H$ with $\sigma$ invertible if and only if $0 \rightarrow A \rightarrow B$ is an $H$-Galois extension having the normal basis property.

In addition, although our crossed products were defined via a left (weak) action of $H$ on $A$, so that $A \#_{\sigma} H$ is a free left $A$-module of rank $\operatorname{dim}_{k} H$, we prove here that such crossed products are also free right $A$-modules of rank $\operatorname{dim}_{k} H$ provided $H$ has bijective antipode.

We then give applications of these characterizations in the case when $\operatorname{dim}_{k} H<\infty$. We first extend the "duality theorem" known for smash products [BM,V] to the case of crossed products; that is, for any crossed product with invertible $\sigma,\left(A \#_{\sigma} H\right) \# H^{*} \simeq A \otimes$ End $_{k} H$. We next improve the Maschke-type results of [BCM] by eliminating the hypothesis that $H$ be cocommutative; in particular, we prove that if $A$ is semisimple Artinian and $H$ is semisimple, then $A \#_{\sigma} H$ is semisimple Artinian. Moreover, if $A$ is semiprimitive (resp. semiprime), $H$ is 
semisimple, and the action of $H$ is inner, then $A \#_{\sigma} H$ is also semiprimitive (resp. semiprime). Finally, we prove a Noether-Skolem theorem for an action of a Hopf algebra on a central simple algebra $B$; as a special case, if the invariants $A=B^{H}$ contain the center of $B, A \subseteq B$ is $H^{*}$-Galois, and $H$ is semisimple, then the action of $H$ is inner on $B$. This special case generalizes the Noether-Skolem theorem for strongly graded rings due to J. Osterburg and D. Quinn [OQ]. An earlier Noether-Skolem theorem for pointed cocommutative Hopf algebras was proved by M. Sweedler [S1].

We assume the reader to be familiar with the basic notions of Hopf algebra theory, including those of convolution, $H$-module algebra, smash product, and right $H$-comodule (see [S2]). Throughout we use Sweedler's "sigma notation." If an algebra $A$ over a field $k$ is a right $H$-comodule via $\rho: A \rightarrow A \otimes H$, then $A$ will be called an $H$-comodule algebra if $\rho$ is an algebra morphism. The comultiplication $\Delta$ of any Hopf algebra $H$ turns $H$ into a right $H$-comodule algebra. Weak actions and coactions and inner actions and coactions are treated in [BCM, $\S \S 1$ and 2]. Finally, if $B$ is an $H$-module algebra, then $B^{H}=$ $\{b \in B: h \cdot b=\varepsilon(1) b$, all $h \in H\}$ is the subalgebra of $H$-invariants; if $B$ is an $H$-comodule algebra, $B^{\operatorname{co} H}=\{b \in B: \rho b=b \otimes 1\}$ is the subalgebra of $H$-coinvariants.

1. A characterization of crossed products. The notion of crossed product of a $k$-algebra $A$ by a Hopf algebra $H$ over $k$ was introduced in [BCM] by the present authors and M. Cohen and in [DT] by Doi and Takeuchi, as follows: Let $H$ act weakly on $A$ [BCM, Definition 1.1] and let $\sigma: H \times H \rightarrow A$ be a $k$-bilinear map. Define a multiplication on $A \otimes H$ by

$$
(a \otimes h)(b \otimes l)=\sum_{(h)(l)} a\left(h_{(1)} \cdot b\right) \sigma\left(h_{(2)}, l_{(1)}\right) \otimes h_{(3)} l_{(2)}
$$

and denote the resulting (possibly non-associative) algebra by $A \#_{\sigma} H$. If $A \#_{\sigma} H$ is associative with $1 \# 1$ as identity element, we call $A \#_{\sigma} H$ a crossed product. A necessary and sufficient condition that $A \#_{\sigma} H$ be a crossed product is that $\sigma$ be normal $(\sigma(h, 1)=\sigma(1, h)=$ $\varepsilon(h) 1)$, that $\sigma$ satisfy the twisted module condition

$$
\begin{aligned}
\sum_{(h)(l)}\left(h_{(1)} \cdot\right. & \left.\left(l_{(1)} \cdot a\right)\right) \sigma\left(h_{(2)}, l_{(2)}\right) \\
& =\sum_{(h)(l)} \sigma\left(h_{(1)}, l_{(1)}\right)\left(h_{(2)} l_{(2)} \cdot a\right),
\end{aligned}
$$


and that it satisfy the cocycle condition

$$
\begin{array}{r}
\sum_{(h)(l)(m)}\left[h_{(1)} \cdot \sigma\left(l_{(1)}, m_{(1)}\right)\right] \sigma\left(h_{(2)}, l_{(2)} m_{(2)}\right) \\
=\sum_{(h)(l)} \sigma\left(h_{(1)}, l_{(1)}\right) \sigma\left(h_{(2)} l_{(2)}, m\right)
\end{array}
$$

for all $h, l, m \in H, a \in A$ [BCM, Corollary 4.6] and [DT, Lemma 10].

Let $B=A \#_{\sigma} H$ be a crossed product and define $\rho: B \rightarrow B \otimes_{k} H$ by

$$
\rho(a \# h)=\sum_{(h)}\left(a \# h_{(1)}\right) \otimes h_{(2)} .
$$

LEMMA 1.5. $\rho$ turns $B$ into a right $H$-comodule algebra and $B^{\mathrm{co} H}=$ $A \#_{\sigma} 1$. Moreover, the map $\gamma: H \rightarrow B$ defined by $\gamma(h)=1 \# h$ is an $H$ comodule map.

Proof. Left to reader. (See [BCM, p. 701 and Lemma 5.10].)

Now $B, A, H$, and $\rho$ as above give us an example of a notion of right $H$-extension, due to Sweedler [S1], Chase and Sweedler [CS], and Kreimer and Takeuchi [KT]:

Definition 1.6. Let $A$ and $B$ be $k$-algebras and let $H$ be a Hopf algebra defined over $k$. Suppose that

(1) $B$ is a right $H$-comodule algebra with structure map $\rho: B \rightarrow$ $B \otimes_{k} H$, and

(2) we are given an algebra injection $l: A \rightarrow B$ such that

(3) $l(A)=B^{\mathrm{co} H}$.

We then say that $0 \rightarrow A \stackrel{l}{\rightarrow} B$ is a right $H$-extension of $A$. For short, we shall sometimes simply refer to the $H$-extension $B / A$, where $l$ is understood. If $A$ is a subalgebra of $B$, we will refer to the $H$-extension $A \subseteq B$. If, moreover, $0 \rightarrow A \stackrel{l}{\rightarrow} B^{\prime}$ is another right $H$-extension of $A$, we say that the two extensions are equivalent if there exists a bijection $\psi: B \rightarrow B^{\prime}$ such that the diagram

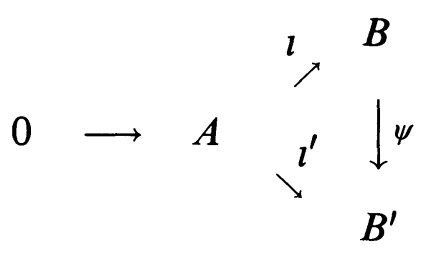

commutes, and $\psi$ is a right $H$-comodule morphism as well as an algebra morphism. 
If $A, H$, and $\sigma$ are as in the beginning of this section with $A \#_{\sigma} H=B$ a crossed product, and if we define $l: A \rightarrow B$ in the obvious way

$$
l(a)=a \# 1,
$$

then $0 \rightarrow A \stackrel{l}{\rightarrow} B$ is a right $H$-extension of $A$.

According to Lemma 1.5, the map $\gamma: H \rightarrow A \#_{\sigma} H$ defined there is an $H$-comodule map. Our first result gives a necessary and sufficient condition that $\gamma$ be convolution invertible. It is a significant strengthening of [BCM, Lemma 6.2], the proof of which required $H$ to be cocommutative.

Proposition 1.8. Let $B=A \#_{\sigma} H$ be a crossed product. Then $\gamma$ is convolution invertible in $\operatorname{Hom}_{k}(H, B)$ if and only if $\sigma$ is convolution invertible in $\operatorname{Hom}_{k}\left(H \otimes_{k} H, A\right)$.

Proof. Using $l$ to identify $A$ with $A \#_{\sigma} 1$, we have

$$
\gamma(h) \gamma(l)=\sum_{(h)(l)} \sigma\left(h_{(1)}, l_{(1)}\right) \gamma\left(h_{(2)} l_{(2)}\right), \quad h, l \in H .
$$

If $\gamma$ is invertible with convolution inverse $\gamma^{-1}$, we have

$$
\sigma(h, l)=\sum_{(h)(l)} \gamma\left(h_{(1)}\right) \gamma\left(l_{(1)}\right) \gamma^{-1}\left(h_{(2)} l_{(2)}\right), \quad h, l \in H,
$$

from which it is obvious that $\sigma$ is invertible with inverse

$$
\sigma^{-1}(h, l)=\sum_{(h)(l)} \gamma\left(h_{(1)} l_{(1)}\right) \gamma^{-1}\left(l_{(2)}\right) \gamma^{-1}\left(h_{(2)}\right) \text {. }
$$

Conversely, suppose $\sigma$ is invertible with inverse $\sigma^{-1}$, and write

$$
\mu(h)=\sum_{(h)} \sigma^{-1}\left(S h_{(2)}, h_{(3)}\right) \# S h_{(1)} .
$$

It was shown in [BCM, Lemma 6.2] that $\mu$ is a left inverse for $\gamma$, that

$$
(\gamma * \mu)(h)=\sum_{(h)}\left[h_{(1)} \cdot \sigma^{-1}\left(S h_{(4)}, h_{(5)}\right)\right] \sigma\left(h_{(2)}, S h_{(3)}\right) \# 1,
$$

and hence that $\mu$ is a right inverse for $\gamma$ if and only if

$$
\sum_{(h)}\left[h_{(1)} \cdot \sigma^{-1}\left(S h_{(4)}, h_{(5)}\right)\right] \sigma\left(h_{(2)}, S h_{(3)}\right)=\varepsilon(h) 1
$$

for all $h \in H$. 
Since $\sigma$ is invertible, the cocycle condition (1.3) gives

$$
\begin{aligned}
h \cdot \sigma(l, m)=\sum_{(h)(l)(m)} \sigma\left(h_{(1)}, l_{(1)}\right) \sigma\left(h_{(2)} l_{(2)}, m_{(1)}\right) \\
\cdot \sigma^{-1}\left(h_{(3)}, l_{(3)} m_{(2)}\right)
\end{aligned}
$$

for all $h, l, m \in H$. Letting $h \in H$ act on the identity

$$
\sum_{(l)(m)} \sigma^{-1}\left(l_{(1)}, m_{(1)}\right) \sigma\left(l_{(2)}, m_{(2)}\right)=\varepsilon(l) \varepsilon(m) 1
$$

we have

$$
\begin{gathered}
\sum_{(h)(l)(m)}\left[h_{(1)} \cdot \sigma^{-1}\left(l_{(1)}, m_{(1)}\right)\right]\left[h_{(2)} \cdot \sigma\left(l_{(2)}, m_{(2)}\right)\right] \\
=\varepsilon(h) \varepsilon(l) \varepsilon(m) 1,
\end{gathered}
$$

that is, $h \otimes l \otimes m \mapsto h \cdot \sigma^{-1}(l, m)$ is the convolution inverse of $h \otimes l \otimes m \mapsto$ $h \cdot \sigma(l, m)$ in $\operatorname{Hom}_{k}(H \otimes H \otimes H, A)$. Hence, from (1.14) we obtain

$$
\begin{aligned}
& h \cdot \sigma^{-1}(l, m)= \sum_{(h)(l)(m)} \sigma\left(h_{(1)}, l_{(1)} m_{(1)}\right) \\
& \cdot \sigma^{-1}\left(h_{(2)} l_{(2)}, m_{(2)}\right) \sigma^{-1}\left(h_{(3)}, l_{(3)}\right) .
\end{aligned}
$$

We may now verify (1.13) using (1.16):

$$
\begin{aligned}
\sum_{(h)} & {\left[h_{(1)} \cdot \sigma^{-1}\left(S h_{(4)}, h_{(5)}\right)\right] \sigma\left(h_{(2)}, S h_{(3)}\right) } \\
& =\sum_{(h)} \sigma\left(h_{(1)},\left(S h_{(8)}\right) h_{(9)}\right) \sigma^{-1}\left(h_{(2)}\left(S h_{(7)}\right), h_{(10)}\right) \\
& \cdot \sigma^{-1}\left(h_{(3)}, S h_{(6)}\right) \sigma\left(h_{(4)}, S h_{(5)}\right) \\
& =\sum_{(h)} \sigma\left(h_{(1)},\left(S h_{(6)}\right) h_{(7)}\right) \sigma^{-1}\left(h_{(2)}\left(S h_{(5)}\right), h_{(8)}\right) \varepsilon\left(h_{(3)}\right) \varepsilon\left(h_{(4)}\right) \\
& =\sum_{(h)} \sigma\left(h_{(1)},\left(S h_{(4)}\right) h_{(5)}\right) \sigma^{-1}\left(h_{(2)}\left(S h_{(3)}\right), h_{(6)}\right) \\
& =\sum_{(h)} \varepsilon\left(h_{(3)}\right) \sigma\left(h_{(1)}, 1\right) \sigma^{-1}\left(1, h_{(4)}\right) \varepsilon\left(h_{(2)}\right)=\varepsilon(h) 1 .
\end{aligned}
$$

Now if $0 \rightarrow A \rightarrow B$ is a right $H$-extension, $B$ becomes an $A$ bimodule in the obvious way. We recall the following definitions:

Definition 1.17. The right $H$-extension $0 \rightarrow A \rightarrow B$

(i) is (right) cleft ([S1, p. 229], [D, p. 1162]) if there exists a right $H$-comodule map $\gamma: H \rightarrow B$ which is invertible in the convolution algebra $\operatorname{Hom}_{k}(H, B)$. 
(ii) is (right) $H$-Galois ([CS, Definition 7.3], [KT, Definition 1.4]) if the map $\beta: B \otimes_{A} B \rightarrow B \otimes_{k} H$ given by $\beta\left(b \otimes_{A} c\right)=(b \otimes 1) \rho(c)$ is bijective.

(iii) has the (right) normal basis property [KT, Definition 2.6] if there is a bijection from $A \otimes_{k} H$ to $B$ which is a left $A$-module and right $H$-comodule morphism.

It is clear that the right $H$-extension $0 \rightarrow A \rightarrow A \#_{\sigma} H$ has the normal basis property if $A \#_{\sigma} H$ is a crossed product, and Proposition 1.8 implies that the extension is cleft if $\sigma$ is invertible.

The following theorem, which combines results of Doi and Takeuchi with Proposition 1.8, ties all of the foregoing notions together.

THeOREM 1.18. Let $0 \rightarrow A \stackrel{l}{\rightarrow} B$ be right $H$-extensions.

Then the following are equivalent:

(i) $0 \rightarrow A \rightarrow B$ is equivalent to $0 \rightarrow A \rightarrow A \#_{\sigma} H$ for some weak action of $H$ on $A$ and some invertible normal cocycle $\sigma$ satisfying the twisted module condition;

(ii) $0 \rightarrow A \rightarrow B$ is cleft;

(iii) $0 \rightarrow A \rightarrow B$ is $H$-Galois and has the normal basis property.

Moreover, if the antipode of $H$ is bijective, then any of the equivalent conditions (i), (ii), or (iii) implies that $B \simeq H \otimes A$ as right $A$-modules.

Proof. [DT, Theorem 9] proves that (ii) and (iii) are equivalent. Suppose (ii) holds. Then there exists an invertible $H$-comodule map $\gamma: H \rightarrow B$ with convolution inverse $\gamma^{-1}$. Since $\gamma(1) \gamma^{-1}(1)=$ $\left(\gamma * \gamma^{-1}\right)(1)=1$ and similarly $\gamma^{-1}(1) \gamma(1)=1$, we see that $\gamma(1)$ is an invertible element in $B$ with inverse $\gamma^{-1}(1)$. According to [D, Lemma 3.2], $\rho\left(\gamma^{-1}(1)\right)=\gamma^{-1}(1) \otimes 1$ so that $\gamma^{-1}(1) \in B^{\mathrm{co} H}$. Define $\tilde{\gamma}(h)=\gamma^{-1}(1) \gamma(h)$ for $h \in H$. It is then easy to check that $\tilde{\gamma}$ is an $H$-comodule map with $\tilde{\gamma}(1)=1$ and with convolution inverse $\tilde{\gamma}^{-1}(h)=\gamma^{-1}(h) \gamma(1)$ for $h \in H$. [DT, Theorem 11] then produces a weak action of $H$ on $B^{\mathrm{co} H}$, a normal cocyle $\sigma^{\prime}$ satisfying the twisted module condition, and an algebra isomorphism $\psi^{\prime}$ of $B^{\operatorname{co} H} \#_{\sigma^{\prime}} H$ onto $B$. These are given, respectively, by

$$
h \cdot b=\sum_{(h)} \tilde{\gamma}\left(h_{(1)}\right) b \tilde{\gamma}^{-1}\left(h_{(2)}\right)
$$

$$
\sigma^{\prime}(h, l)=\sum_{(h)(l)} \tilde{\gamma}\left(h_{(1)}\right) \tilde{\gamma}\left(l_{(1)}\right) \tilde{\gamma}^{-1}\left(h_{(2)} l_{(2)}\right)
$$


and

$$
\psi^{\prime}\left(b \#_{\sigma^{\prime}} h\right)=b \tilde{\gamma}(h)
$$

for $b \in B^{\mathrm{co} H}, h, l \in H$. As in Proposition 1.8, it is clear that $\sigma^{\prime}$ is invertible. From $(1.21)$ it is equally clear that $\psi^{\prime}$ is an $H$-comodule map, since $\tilde{\gamma}$ is. Therefore the $H$-extensions $0 \rightarrow B^{\operatorname{co} H} \rightarrow B$ and $0 \rightarrow$ $B^{\mathrm{co} H} \rightarrow B^{\mathrm{co} H} \#_{\sigma^{\prime}} H$ are equivalent. Using the algebra isomorphism $l$ to transport the weak action of $H$ and the cocycle $\sigma^{\prime}$ over to $A$, we obtain a weak action of $H$ on $A$, an $A$-valued invertible cocycle $\sigma$ and a map $\psi: A \#_{\sigma} H \rightarrow B$ setting up an equivalence of $0 \rightarrow A \stackrel{l}{\rightarrow} B$ with $0 \rightarrow A \rightarrow A \#_{\sigma} H$. Thus (i) holds. Finally if (i) holds, $0 \rightarrow A \stackrel{l}{\rightarrow} B$ is cleft because $0 \rightarrow A \rightarrow A \#_{\sigma} H$ is by Proposition 1.8. Therefore (ii) holds.

Finally we prove that $B \cong H \otimes A$ as right $A$-modules, provided the antipode $S$ of $H$ is bijective and $0 \rightarrow A \rightarrow B$ is cleft. Thus let $\bar{S}$ denote the composition inverse of $S$ and let $\gamma: H \rightarrow B$ be an invertible right $H$-comodule map. Then [DT, Proposition 8] tells us that $\mu=\gamma^{-1} \circ \bar{S}$ is a right $H$-comodule map from $H$ to $B$ and that, if we set $\hat{\mu}=\gamma \circ \bar{S}$, we have

$$
\sum_{(h)} \mu\left(h_{(2)}\right) \hat{\mu}\left(h_{(1)}\right)=\sum_{(h)} \hat{\mu}\left(h_{(2)}\right) \mu\left(h_{(1)}\right)=\varepsilon(h) 1
$$

for all $h \in H$. Moreover it follows immediately from [D, Lemma 3.2] that $\hat{\mu}$ satisfies

$$
\rho(\hat{\mu}(h))=\sum_{(h)} \hat{\mu}\left(h_{(2)}\right) \otimes \bar{S} h_{(1)}, \quad h \in H .
$$

Define $\alpha: H \otimes_{k} A \rightarrow B$ by $\alpha(h \otimes a)=\mu(h)(l a)$ for $h \in H, a \in A$. We next note that

$$
\begin{aligned}
\rho \sum_{(b)} \hat{\mu}\left(b_{(1)}\right) b_{(0)} & =\sum_{(b)} \hat{\mu}\left(b_{(3)}\right) b_{(0)} \otimes\left(\bar{S} b_{(2)}\right) b_{(1)} \\
& =\left(\sum_{(b)} \hat{\mu}\left(b_{(1)}\right) b_{(0)}\right) \otimes 1
\end{aligned}
$$

for $b \in B$, so that $\sum_{(b)} \hat{\mu}\left(b_{(1)}\right) b_{(0)} \in B^{\mathrm{co} H}$. Thus we may define $P(b)=l^{-1}\left(\sum_{(b)} \hat{\mu}\left(b_{(1)}\right) b_{(0)}\right)$ for $b \in B$, and $P: B \rightarrow A$. Furthermore $P(b(l a))=P(b) a$ for $b \in B, a \in A$. Now define $\bar{\alpha}: B \rightarrow H \otimes_{k} A$ by $\bar{\alpha}(b)=\sum_{(b)} b_{(1)} \otimes P\left(b_{(0)}\right)$. Both $\alpha$ and $\bar{\alpha}$ are right $A$-module maps. Now

$$
\sum_{(\mu(h))} \hat{\mu}\left(\mu(h)_{(1)}\right) \mu(h)_{(0)}=\sum_{(h)} \hat{\mu}\left(h_{(2)}\right) \mu\left(h_{(1)}\right)=\varepsilon(h) 1
$$


so that $P(\mu(h))=\varepsilon(h) 1$ for all $h \in H$. It follows that

$$
\bar{\alpha}(\alpha(h \otimes 1))=\bar{\alpha}(\mu(h))=\sum_{(h)} h_{(2)} \otimes P\left(\mu\left(h_{(1)}\right)\right)=h \otimes 1
$$

and so $\bar{\alpha} \circ \alpha=$ id. And $\alpha \circ \bar{\alpha}=$ id follows immediately from (1.22). Thus $\alpha: H \otimes_{k} A \rightarrow B$ is a right $A$-module isomorphism.

REMARK 1.24. The justification for calling extensions satisfying Definition 1.17 (ii) Galois may be found in [CS, pp. 54-60] and [CHR, Remarks 1.5]. However, why extensions satisfying Definition 1.17 (iii) should be said to have the normal basis property needs further explanation: Suppose $\operatorname{dim}_{k} H<\infty$. Then by results of Larson and Sweedler (see [S2, Theorem 5.1.3 and Corollary 5.1.6]) we have that $H$ contains a non-zero left integral $x$ (i.e. $h x=\varepsilon(h) x$ for all $h \in H)$ and that the map

$$
f \mapsto(f-x), \quad f \in H^{*}
$$

maps $H^{*}$ bijectively onto $H$ and so maps $k$-bases of $H^{*}$ to $k$-bases of $H$. Now let $\varphi: A \otimes_{k} H \rightarrow B$ be a bijective left $A$-module and right $H$-comodule morphism. Then it is also a left $H^{*}$-module morphism, where as usual $f \cdot(a \otimes h)=a \otimes f \rightarrow h$ and $f \cdot b=\sum_{(b)}\left\langle f, b_{(1)}\right\rangle b_{(0)}$. Let $u=\varphi(1 \otimes x)$. Then it follows that if $\left\{f_{1}, \ldots, f_{n}\right\}$ is a $k$-basis for $H^{*},\left\{1 \otimes\left(f_{1} \rightarrow x\right), \ldots, 1 \otimes\left(f_{n}-x\right)\right\}$ is an $A$-basis for $A \otimes_{k} H$, and so $\left\{f_{1} \cdot u, \ldots, f_{n} \cdot u\right\}$ is a basis for the free left $A$-module $B$. Conversely, suppose that for some $0 \neq u \in B,\left\{f_{1} \cdot u, \ldots, f_{n} \cdot u\right\}$ is a basis for the free left $A$-module $B$. We may then define $\varphi: A \otimes_{k} H \rightarrow B$ by $\varphi(a \otimes(f-x))=a(f \cdot u)$ since $(1.25)$ is bijective. It follows that $\varphi$ is a bijection and is a left $A$-module map. Since it is a left $H^{*}$-module map, it must be a right $H$-comodule map. So for finite dimensional $H,(1.17$ (iii)) corresponds exactly to the idea of a normal basis. Note however that if $\operatorname{dim}_{k} H=\infty,(1.25)$ cannot be a surjection for any $x \in H$.

EXAMPLE 1.26. Let $B$ be a field, $k \subseteq B$, and let $G$ be a finite subgroup of $\operatorname{Gal}(B / k)$. Let $A=B^{G}$. If we set $H=(k G)^{*}$, then $H^{*}=k G . \quad B$ is a left $H^{*}$-module algebra. Since $\operatorname{dim}_{k} H<\infty$, the left $H^{*}$-action is associated to a right $H$-coaction and $A=B^{\mathrm{co} H}$. Let $\left\{p_{x}: x \in G\right\}$ be the $k$-basis of $H$ dual to the basis $\{x: x \in G\}$ of $H^{*}$. Then the coaction $\rho$ of $H$ on $B$ is given by

$$
\rho b=\sum_{x \in G}(x \cdot b) \otimes p_{x}, \quad b \in B .
$$


Since $B$ is a Galois extension of $A$ with a normal basis in the ordinary sense, the left $H$-extension $A \subseteq B$ is Galois with the normal basis property in the sense of Definition 1.17 by [CHR, Theorem 1.3 and Remark 1.5 (a)] and Remark 1.24 above. Thus by Theorem 1.18, the extension $0 \rightarrow A \rightarrow B$ is equivalent to $0 \rightarrow A \rightarrow A \#_{\sigma} H$ for some weak action and some cocycle $\sigma$. Since $B$ is commutative, (1.19) says that the weak action is trivial. We now compute $\sigma$. Choose $0 \neq u \in B$ so that $\{x \cdot u: x \in G\}$ is an $A$-basis for $B$. Clearly $0 \neq c=\sum_{x \in G} x \cdot u \in A$. Dividing by $c$ we may assume that $\sum_{x \in G} x \cdot u=1$. The map $\gamma: H \rightarrow B$ of Definition 1.17 (i) is related to the normal basis isomorphism $\varphi$ of Remark 1.24 via $\gamma(h)=\varphi(1 \otimes h)$ for $h \in H$. Now $p_{e}$ is a left integral in $H$ [S2, p. 92]. Thus $\gamma\left(p_{x}\right)=\varphi\left(1 \otimes\left(x^{-1} \rightarrow p_{e}\right)\right)=x^{-1} \cdot u$ for all $x \in G$. Since $\gamma(1)=\sum_{x \in G} \gamma\left(p_{x}\right)=1$, we see that $\tilde{\gamma}=\gamma$ in (1.20). Therefore the cocycle is determined by the equations

$$
\begin{aligned}
\left(x^{-1} \cdot u\right)\left(y^{-1} \cdot u\right)=\gamma\left(p_{x}\right) \gamma\left(p_{y}\right) & \\
& =\sum_{z, w \in G} \sigma\left(p_{x z^{-1}}, p_{y w^{-1}}\right) \gamma\left(p_{z} p_{w}\right) \\
& =\sum_{z \in G} \sigma\left(p_{x z^{-1}}, p_{y z^{-1}}\right) \gamma\left(p_{z}\right) \\
& =\sum_{z \in G} \sigma\left(p_{x z^{-1}}, p_{y z^{-1}}\right)\left(z^{-1} \cdot u\right), \quad \text { or better, }
\end{aligned}
$$

$$
(x \cdot u)(y \cdot u)=\sum_{z \in G} \sigma\left(p_{x^{-1} z}, p_{y^{-1} z}\right)(z \cdot u) .
$$

Equations (1.29) are solvable. In fact, the structure constants $a_{x, y}^{z} \in A$ of $B / A$ with respect to the normal basis $\{x \cdot u: x \in G\}$, that is, the solutions of $(x \cdot u)(y \cdot u)=\sum_{z \in G} a_{x, y}^{z}(z \cdot u)$, satisfy the condition $a_{x, y}^{z}=a_{z^{-1} x, z^{-1} y}^{e}$ because the $x \in G$ are automorphisms of $B / A$. So we can set $\sigma\left(p_{x}, p_{y}\right)=a_{x^{-1}, y^{-1}}^{e}$. This shows that every finite Galois field extension $B / A$ is a twisted product in the sense of [BCM, Example 4.10] of $A$ with $(k \operatorname{Gal}(B / A))^{*}$.

REMARK 1.30. Theorem 4.14 of [BCM] is a special case of Theorem $1.18((\mathrm{ii}) \Rightarrow(\mathrm{i}))$, or more, precisely, of [DT, Theorem 11]: Let $H \stackrel{\pi}{\rightarrow} \bar{H} \rightarrow 0$ be an exact sequence of Hopf algebras and let $\gamma: \bar{H} \rightarrow H$ be a coalgebra splitting of $\pi$ such that $\gamma(\overline{1})=1$. Then [BCM, Theorem 4.14] says that $H$ is algebra isomorphic to $A \#_{\sigma} \bar{H}$, where $A=\operatorname{LHKer} \pi=\operatorname{Ker}(\mathrm{id} \otimes \pi) \circ \Delta$, for a suitable weak action of $\bar{H}$ on $A$ and a suitable cocycle $\sigma$. We obtain this from Theorem 11 
of [DT] by noting that $H$ is a right $\bar{H}$-comodule algebra via the map $\rho=(\mathrm{id} \otimes \pi) \circ \Delta$ and LHKer $\pi=H^{\mathrm{co} \bar{H}}$. Moreover, $\gamma$ is a $\bar{H}$-comodule map since $\rho \circ \gamma=(\mathrm{id} \otimes \pi) \circ \Delta \circ \gamma=(\mathrm{id} \otimes \pi) \circ(\gamma \otimes \gamma) \circ \Delta=(\gamma \otimes \mathrm{id}) \circ \Delta$, and $\gamma^{-1}=S \circ \gamma$ since $\gamma$ is a coalgebra map. Thus Theorem 11 of [DT] applies, giving the conclusion of Theorem 4.14 of [BCM]. We note that the proof of the theorem in [BCM] is virtually identical to that of [DT].

However, in the situation of an exact sequence $H \stackrel{\pi}{\rightarrow} \bar{H} \rightarrow 0$ of Hopf algebras, [DT, Theorem 11] is actually stronger than [BCM, Theorem 4.14]: the exact sequence may have no coalgebra splitting, yet the $\bar{H}$ extension $0 \rightarrow$ LHKer $\pi \rightarrow H$ may be cleft. In fact, let $G$ be any finite group and let $L$ be a subgroup of $G$. Set $H=(k G)^{*}$ and $\bar{H}=(k L)^{*}$. The inclusion map $L \hookrightarrow G$ induces a surjection $\pi: H \rightarrow \bar{H}$. Define the $\bar{H}$-coaction on $H$ by $\rho=(\mathrm{id} \otimes \pi) \circ \Delta$ as above. Using the notation of Example 1.26, we write $p_{x} \in H$ for $x \in G$ and $\bar{p}_{y} \in \bar{H}$ for $y \in L$. Let $S$ be a set of coset representatives for $G / L$ so that $G=S L$. Define $\gamma: \bar{H} \rightarrow H$ by $\gamma\left(\bar{p}_{y}\right)=\sum_{x \in S} p_{x y}$ for $y \in L$. Then $\gamma$ is an $\bar{H}$-comodule map with convolution inverse $\gamma^{-1}$ given by $\gamma^{-1}\left(\bar{p}_{y}\right)=\sum_{x \in S} p_{x y^{-1}}$, and $\gamma(1)=\overline{1}$. So this extension is always cleft. But if $k=\mathbf{C}$ and we take $G$ to be $S_{3}$ and let $L$ be $\langle(1,2,3)\rangle$, there is no coalgebra map from $\bar{H}$ to $H$ splitting $\pi$ since there is no surjective algebra morphism from $H^{*}=\mathrm{C} G$ to $\bar{H}^{*}=\mathrm{C} L$ (see $\left.[\mathrm{M}]\right)$.

REMARK 1.31. We can also define left $H$-extensions and the properties of being left cleft, left $H$-Galois, and having the left normal basis property. Thus, $\gamma$ in Definition 1.17 (i) would be a left $H$-comodule map, $\beta$ in Definition 1.17 (ii) would be defined by $\beta\left(b \otimes_{A} c\right)=$ $\sum_{(b)} b_{(-1)} \otimes b_{(0)} c$, and the bijection in Definition 1.17 (iii) would be a right $A$-module, left $H$-comodule map from $H \otimes_{k} A$ to $B$. We can also define a crossed product $H_{\sigma} \# A$ from a right weak action of $H$ on $A$ by replacing (1.1) with

$$
(h \otimes a)(l \otimes b)=\sum_{(h)(l)} h_{(1)} l_{(1)} \otimes \sigma\left(h_{(2)}, l_{(2)}\right)\left(a \cdot l_{(3)}\right) b .
$$

Then Lemma 1.5, Proposition 1.8, and Theorem 1.18 all go through in this setting.

2. Applications: finite dimensional Hopf algebras. When $H$ is finite dimensional, Proposition 1.8 and Theorem 1.18 can be used to obtain a duality theorem, some Maschke-type results, and a Noether-Skolem theorem.

We assume throughout $\S 2$ that $\operatorname{dim}_{k} H<\infty$. 
A. Duality. A crossed product $A \#_{\sigma} H$ is an $H^{*}$-module algebra via the formula

$$
f \cdot(a \# h)=a \#(f-h)=\sum_{(h)}\left\langle f, h_{(2)}\right\rangle a \# h_{(1)}
$$

for $a \in A, h \in H, f \in H^{*}$. Thus we may form the smash product $\left(A \#_{\sigma} H\right) \# H^{*}$. When $\sigma$ is trivial, that is, when $A \#_{\sigma} H=A \# H$, an ordinary smash product, the present authors proved in [BM] that $\left(A \#_{\sigma} H\right) \# H^{*}$ is algebra isomorphic to $A \otimes_{k}\left(H \# H^{*}\right)$ under certain hypotheses on the action of $H$ on $A$. This is the duality theorem. These hypotheses automatically hold if $\operatorname{dim}_{k} H<\infty$, in which case we also have $H \# H^{*} \simeq \operatorname{End}_{k} H$. Independently, in the finite dimensional case with trivial $\sigma$, proofs were given by Van den Bergh [V] and by Koppinen (unpublished). In [BCM, Proposition 5.6], it was observed that the duality theorem held for arbitrary $\sigma$ when $H=k G, G$ a finite group. Here we prove the duality theorem for invertible $\sigma$ and for arbitrary finite dimensional $H$.

Theorem 2.2. Let $\operatorname{dim}_{k} H=n<\infty$ and let $A \#_{\sigma} H$ be a crossed product with invertible cocycle $\sigma$. Then

$$
\left(A \# \#_{\sigma} H\right) \# H^{*} \simeq A \otimes \operatorname{End}_{k} H \simeq M_{n}(A)
$$

Proof. By Theorem 1.18, $0 \rightarrow A \stackrel{l}{\rightarrow} A \#_{\sigma} H=B$ is $H$-Galois and has the normal basis property. By [KT, Theorem 1.7], $B \# H^{*}$ is algebra isomorphic to $\operatorname{End}_{A}^{r}(B)$, the right $A$-linear morphisms from $B$ to $B$. The antipode $S$ of $H$ is bijective by [S2, p. 101]. So Theorem 1.18 says that $B \simeq H \otimes A$, as right $A$-modules, and we have $\operatorname{End}_{A}^{r}(B) \simeq$ $M_{n}(A)$.

B. Maschke-type theorems. In [LS, Proposition 3], Maschke's classical theorem on semisimplicity of group algebras was extended to Hopf algebras: a finite dimensional Hopf algebra $H$ is semisimple if and only if $\varepsilon(x) \neq 0$ for $x$ a nonzero left integral in $H$. Their method was to prove that every left $H$-module is completely reducible via an averaging argument which extends the classical Maschke proof.

In [CF, Theorem 4] this argument was extended to smash products $A \# H$ by showing that the [LS] averaging map was also a left $A$-module map. A partial extension to crossed products $A \#_{\sigma} H$ was proved in [BCM, Corollary 6.6] under the additional hypotheses that 
$\sigma$ is invertible and $H$ is cocommutative. In this subsection we improve results in (6.3-6.10) of [BCM] and, in particular, remove the cocommutativity hypothesis in the [BCM] Maschke theorem.

First, our improvement of [BCM, Lemma 6.3]:

LEMMA 2.3. Let $A \#_{\sigma} H$ be a crossed product with invertible $\sigma$ and finite dimensional $H$, and let $x$ be a left integral in $H$. Let $V, W$ be left $A \#_{\sigma} H$-modules and let $\lambda \in \operatorname{Hom}_{A \#_{\sigma} 1}^{l}(V, W)$. Define $\tilde{\lambda}: V \rightarrow W$ by

$$
\tilde{\lambda}(v)=\sum_{(x)} \gamma^{-1}\left(x_{(1)}\right) \cdot \lambda\left(\gamma\left(x_{(2)}\right) v\right)
$$

for all $v \in V$. Then $\tilde{\lambda} \in \operatorname{Hom}_{A \#_{\sigma} H}^{l}(V, W)$.

Proof. Note that $\gamma^{-1}$ exists by Proposition 1.8 so that (2.4) makes sense. We identify $A$ with $A \#_{\sigma} 1$. Then $\tilde{\lambda}$ is a left $A$-module map by the same argument as in [BCM, Lemma 6.3], which only requires the invertibility of $\gamma$. Similar to the argument on [BCM, p. 707], we have

$$
h \otimes \Delta x=\sum_{(h)(x)} h_{(1)} \otimes x_{(1)} h_{(2)} \otimes x_{(2)} h_{(3)}, \quad h \in H .
$$

Now let $h \in H, v \in V$. Then

$$
\begin{aligned}
\tilde{\lambda}(\gamma(h) v) & =\sum_{(x)} \gamma^{-1}\left(x_{(1)}\right) \cdot \lambda\left(\gamma\left(x_{(2)}\right) \gamma(h) v\right) \\
& =\sum_{(x)(h)} \gamma^{-1}\left(x_{(1)}\right) \cdot \lambda\left(\sigma\left(x_{(2)}, h_{(1)}\right) \gamma\left(x_{(3)} h_{(2)}\right) v\right) \\
& =\sum_{(x)(h)} \gamma^{-1}\left(x_{(1)}\right) \sigma\left(x_{(2)}, h_{(1)}\right) \cdot \lambda\left(\gamma\left(x_{(3)} h_{(2)}\right) v\right) \\
& =\sum_{(x)(h)} \gamma\left(h_{(1)}\right) \gamma^{-1}\left(x_{(1)} h_{(2)}\right) \cdot \lambda\left(\gamma\left(x_{(2)} h_{(3)}\right) v\right) \\
& =\sum_{(x)} \gamma(h) \gamma^{-1}\left(x_{(1)}\right) \cdot \lambda\left(\gamma\left(x_{(2)}\right) v\right)=\gamma(h) \tilde{\lambda}(v),
\end{aligned}
$$

where the second equality follows from (1.9), the third from the fact that $\tilde{\gamma}$ is an $A$-module map, the fourth from (1.10), and the fifth from (2.5). Since $a \# h=a \gamma(h), \tilde{\lambda}$ is an $A \#_{\sigma} H$-module map.

We next remove the cocommutativity hypothesis from [BCM, Theorem 6.4 and Corollary 6.5] obtaining our version of Maschke's theorem. 
THEOREM 2.6. Let $H$ be a finite dimensional semisimple Hopf algebra, and let $A \#_{\sigma} H$ be a crossed product with $\sigma$ invertible.

(1) Let $V$ be a left $A \#_{\sigma} H$-module. If $W \subseteq V$ is a left $A \#_{\sigma} H$ submodule of $V$ which has an A-complement in $V$, then $W$ has an $A \#_{\sigma} H$-complement in $V$. Consequently, if $V$ is completely reducible as a left A-module, it is completely reducible as a left $A \#_{\sigma} H$-module.

(2) If $A$ is semisimple Artinian, so is $A \#_{\sigma} H$.

Proof. The proofs in [BCM] work here if we use Lemma 2.3 instead of [BCM, Lemma 6.3].

The last result of this subsection improves [BCM, Corollary 6.6 and Theorem 6.10].

THeOREM 2.7. Let $H$ be a finite dimensional semisimple Hopf algebra, and let $A \#_{\sigma} H$ be a crossed product with invertible $\sigma$ such that the weak action of $H$ on $A$ is inner. If $A$ is semiprimitive (resp. semiprime), then so is $A \#_{\sigma} H$.

Proof. Since the (weak) action of $H$ on $A$ is inner, [BCM, Theorem 5.3] implies that $A \#_{\sigma} H$ is algebra isomorphic to a twisted product $A_{\tau}[H]$, where $\tau$ is invertible. That is, the action of $H$ can be trivialized by changing cocycles; this means that we may assume that the elements of $A$ commute with the elements of $H$. Now a standard induced module argument shows the semiprimitive part of the theorem: follow the proof of [BCM, Corollary 6.6, case (2)], substituting Theorem 2.6 for [BCM, Corollary 6.5]. Then the semiprime part of the theorem follows exactly as in the proof of [BCM, Theorem 6.10], using the Lorenz-Passman "primitivity machine" and substituting the above semiprimitive result for [BCM, Corollary 6.6].

REMARK 2.8. The question as to whether Theorem 2.7 is true without the inner hypothesis remains open. The method of induced modules does not extend, as noted in [BCM, Example 6.7]; it is false in general that if $V$ is a completely reducible $A$-module, then $\bar{V}=$ $\left(A \#_{\sigma} H\right) \otimes_{A} V$ is completely reducible as an $A$-module, even if $\sigma$ is trivial. We note however that if $H=(k G)^{*}, G$ a finite group, then $A$ semiprime implies $A \#_{\sigma} H$ semiprime even if the weak action is not inner. For if $A \#_{\sigma} H$ contains a non-zero nilpotent ideal $N$, then $x \cdot N$ is also a nilpotent ideal for all $x \in G$, where $\cdot$ is the usual action of $H^{*}=k G$ on $A \#_{\sigma} H$, and so $M=\sum_{x \in G} x \cdot N$ is nilpotent. Since $M$ is 
$G$-stable, $M \# k G$ is a nilpotent ideal of $\left(A \# \#_{\sigma} H\right) \# H^{*} \simeq M_{n}(A)$. But $A$ semiprime implies $M_{n}(A)$ semiprime, a contradiction.

C. A Noether-Skolem theorem. In [S1, Theorem 9.5], Sweedler proved a Noether-Skolem theorem for actions of pointed Hopf algebras $H$, i.e., $H$ having only one-dimensional minimal subcoalgebras. When $k$ is algebraically closed, this includes all cocommutative $H$ and hence the known cases of actions by groups of automorphisms (the classical Noether-Skolem theorem) and by Lie algebras of derivations (a theorem of Jacobson).

For non-cocommutative $H$, the first progress was made recently by Osterburg and Quinn [OQ] who considered the case of an action by $H=(k G)^{*}, G$ a finite group, that is, the case of a $G$-graded algebra $B$ over $k$. They also assumed that $B$ is strongly graded by $G$. By a result of Ulbrich [U1, Lemma 2.1], this assumption is equivalent to $A \subseteq B$ being a right $H^{*}$-Galois extension, where $A=B^{H}=B_{e}$, the identity component of $B$ under the grading.

In this subsection, we will extend the [OQ] result to right $H$-Galois extensions $A \subseteq B$ for arbitrary finite dimensional Hopf algebras $H$.

We first need a lemma which will guarantee that certain Galois extensions have the normal basis property. It extends [KC, Proposition 2].

LemMA 2.9. Let $A \subseteq B$ be a right $H$-Galois extension, $\operatorname{dim}_{k} H<\infty$. Suppose that $A$ is a field contained in the center of $B$. Then $A \subseteq B$ has the (right) normal basis property.

Proof. We must show that $B$ is left $A$-module, right $H$-comodule isomorphic to $A \otimes_{k} H$. Since $\operatorname{dim}_{k} H<\infty$, right $H$-comodule isomorphism is the same as left $H^{*}$-module isomorphism. Since $A=$ $B^{\operatorname{co} H}=B^{H^{*}}$, the left actions of $H^{*}$ and $A$ commute. Thus $A \subseteq B$ has the normal basis property if and only if $B \simeq A \otimes H$ as left $A \otimes H^{*}$ modules.

Since $A \subseteq B$ is (right) $H$-Galois, $B \otimes_{A} B \simeq B \otimes_{k} H$ as left $A \otimes H^{*}$ modules. Since $B \otimes_{k} H \simeq B \otimes_{A}\left(A \otimes_{k} H\right)$, we have

$$
B \otimes_{A} B \simeq B \otimes_{A}\left(A \otimes_{k} H\right)
$$

as left $A \otimes H^{*}$-modules. Now $B$ is a finitely-generated projective right $A$-module because $A \subseteq B$ is $H$-Galois [KT, Theorem 1.7]. Since $A$ is a field central in $B$, it follows that $B \simeq A^{n}$ for some $n$ both as left 
and right $A$-modules. Substituting in the left factor on both sides of (2.10), we obtain

$$
B^{n} \simeq\left(A \otimes_{k} H\right)^{n}
$$

as left $A \otimes H^{*}$-modules. Again using [ $\left.B: A\right]=n$, it follows that $B$ satisfies both the ascending and descending chain conditions on $A \otimes H^{*}$ submodules. Now apply the Krull-Schmidt theorem: by uniqueness of decomposition into direct sums of indecomposable submodules, we must have $B \simeq A \otimes_{k} H$ as left $A \otimes H^{*}$-modules.

The left version of Lemma 2.9 also holds (see Remark 1.31).

We next require some results of K.-H. Ulbrich [U2]. Let $A \subseteq B$ be a right $H$-Galois extension and let $E$ denote the centralizer of $A$ in $B$. There is a right $H$-action on $E$ defined as follows: Let $h \in H$ and write $\beta^{-1}(1 \otimes h)=\sum_{i} b_{i} \otimes_{A} c_{i}, \beta$ as in Definition 1.17 (ii). Let $e \in E$. We set

$$
e-h=\sum_{i} b_{i} e c_{i}
$$

a formula which makes sense because $e$ centralizes $A$. Ulbrich shows that $\leftarrow$ turns $E$ into a right $H$-module algebra and hence, since $\operatorname{dim}_{k} H<\infty$, into a left $H^{*}$-comodule algebra. The right action $<$ is completely characterized by

$$
e b=\sum_{(b)} b_{(0)}\left(e-b_{(1)}\right) \quad \text { for all } e \in E, b \in B
$$

where $\rho: b \mapsto \sum_{(b)} b_{(0)} \otimes b_{(1)} \in B \otimes H$ is the original $H$-coaction on $B$. The subalgebra $E^{H}=E^{\mathrm{co} H^{*}}$ is simply $Z$, the center of $B$ [U2, Lemma 2.1]. We call this action the Miyashita-Ulbrich action, following a suggestion of Doi and Takeuchi.

The next result is part of [ $\mathbf{U} 2$, Satz 2.7], restated in our notation:

Theorem 2.14 (Ulbrich). Let $A \subseteq B$ be a right $H$-Galois extension, and assume that $A$ is a left $A$-module direct summand of $B$. If $B$ is separable over $Z$ and $Z \subseteq A$, then $Z \subseteq E$ is a left $H^{*}$-Galois extension (see Remark 1.31).

We can now prove our Noether-Skolem result. 
THEOREM 2.15. Let $B$ be a central simple algebra with center $Z \supseteq k \cdot 1$. Let $H$ be a finite dimensional Hopf algebra acting (on the left) on $B$ with $A=B^{H} \supseteq Z$. Assume that

(1) $A \subseteq B$ is a right $H^{*}$-Galois extension and

(2) $A$ is a left $A$-module direct summand of $B$.

Then the action of $H$ on $B$ is inner.

Proof. We must find a convolution invertible $u: H \rightarrow B$ such that

$$
h \cdot b=\sum_{(h)} u\left(h_{(1)}\right) b u^{-1}\left(h_{(2)}\right) \quad \text { for all } h \in H, b \in B .
$$

Note that (1) and (2) are just the hypotheses of Ulbrich's theorem with $H$ and $H^{*}$ interchanged. Thus $Z \subseteq E$, the centralizer of $A$ in $B$, is left $H$-Galois, with the right $H^{*}$-action as in (2.12).

Since $Z$ is a field central in $E$, the left version of Lemma 2.9 implies that $Z \subseteq E$ satisfies the left normal basis property of Remark 1.31. Then by the left version of Theorem 1.18 (or [DT, Theorem 9]), $Z \subseteq E$ is left cleft: there exists a left $H$-comodule map $u: H \rightarrow E$ which is convolution invertible in $\operatorname{Hom}_{k}(H, E)$. Hence $u$ is a right $H^{*}$-module map. This is the map $u$ that satisfies (2.16). In fact, let $h \in H$ and $b \in B$. We have

$$
\begin{aligned}
u(h) b & =\sum_{(b)} b_{(0)}\left(u(h)-b_{(1)}\right)=\sum_{(b)} b_{(0)} u\left(h-b_{(1)}\right) \\
& =\sum_{(b)} b_{(0)} u\left(\sum_{(h)}\left\langle b_{(1)}, h_{(1)}\right\rangle h_{(2)}\right) \\
& =\sum_{(b)(h)}\left\langle b_{(1)}, h_{(1)}\right\rangle b_{(0)} u\left(h_{(2)}\right)=\sum_{(h)}\left(h_{(1)} \cdot b\right) u\left(h_{(2)}\right),
\end{aligned}
$$

where the first equality is (2.13), the second holds since $u$ is a right $H^{*}$-module map, the third holds by the definition of the right action $\leftarrow$ of $H^{*}$ on $H$, and the fifth expresses the relation between the right $H^{*}$-coaction and the left $H$-action on $B$. Since $u$ is invertible, we get (2.16).

Note that by Theorem $1.18, E \cong H_{\sigma} \# Z$, a right crossed product over $Z$. Thus we may view the map $u: H \rightarrow E$ in the above proof as $u(h)=\varphi^{-1}(h \otimes 1)$, where $\varphi: E \rightarrow H_{\sigma} \# Z$ is the isomorphism.

REMARK 2.17. Hypothesis (2) of Theorem 2.15 will always be satisfied if $H$ is semisimple. For then $H$ contains a left integral $x$ 
with $\varepsilon(x)=1$. The mapping $t: B \rightarrow A$ given by $t(b)=x \cdot b$ is then an $A$-module projection of $B$ onto $A$ so that $A$ is an $A$-module direct summand of $B$.

Finally we obtain the Osterburg-Quinn result as a consequence of Theorem 2.15.

COROLlARY 2.18. Let $B$ be a central simple algebra with center $Z$. Assume that $B$ is strongly graded by the finite group $G$ (that is, $B=\sum_{x \in G} \oplus B_{x}$ and $B_{x} B_{y}=B_{x y}$ for all $\left.x, y \in G\right)$ and that $Z \subseteq B_{e}$, the identity component of $B$. Then the grading is inner, considering the grading as an action of $(Z G)^{*}$ on $B$ via $f \cdot b=\sum_{x \in G}\langle f, x\rangle b_{x}$, where $b=\sum_{x \in G} b_{x}$ and $b_{x} \in B_{x}$.

Proof. By [U1, Lemma 2.1], $B_{e} \subseteq B$ is right $Z G$-Galois since $B$ is strongly graded, giving hypothesis (1) of Theorem 2.15. Hypothesis (2) holds by Remark 2.17 , since $(Z G)^{*}$ is semisimple.

\section{REFERENCES}

[BCM] R. J. Blattner, M. Cohen and S. Montgomery, Crossed products and inner actions of Hopf algebras, Trans. Amer. Math. Soc., 298 (1986), 671-711.

[BM] R. J. Blattner and S. Montgomery, A duality theorem for Hopf module algebras, J. Algebra, 95 (1985), 153-172.

[CHR] S. U. Chase, D. K. Harrison and A. Rosenberg, Galois theory and Galois cohomology of commutative rings, Mem. Amer. Math. Soc., 52 (1965), 1533.

[CS] S. U. Chase and M. E. Sweedler, Hopf algebras and Galois theory, Lecture Notes in Math., vol. 97, Springer-Verlag, Berlin and New York, 1969.

[CF] M. Cohen and D. Fishman, Hopf algebra actions, J. Algebra, 100 (1986), 363-379.

[D] Y. Doi, Cleft comodule algebras and Hopf modules, Comm. Algebra, 12 (1984), 1155-1169.

[DT] Y. Doi and M. Takeuchi, Cleft comodule algebras for a bialgebra, Comm. Algebra, 14 (1986), 801-817.

[KC] H. F. Kreimer and P. M. Cook II, Galois theories and normal bases, J. Algebra, 43 (1976), 115-121.

[KT] H. F. Kreimer and M. Takeuchi, Hopf algebras and Galois extensions of an algebra, Indiana Math J., 30 (1981), 675-692.

[LS] R. Larson and M. E. Sweedler, An associative orthogonal bilinear form for Hopf algebras, Amer. J. Math., 91 (1969), 75-94.

[M] S. Montgomery, Crossed products of Hopf algebras and enveloping algebras, in Perspectives in Ring Theory, Proceedings of the 1987 NATO workshop in Antwerp, Reidel, to appear.

[OQ] J. Osterburg and D. Quinn, A Noether-Skolem theorem for group graded rings, J. Algebra, 113 (1988), 483-490; addendum, to appear. 
[S1] M. E. Sweedler, Cohomology of algebras over Hopf algebras, Trans. Amer. Math. Soc., 133 (1968), 205-239.

[S2] Hopf Algebras, Benjamin, New York, 1969.

[U1] K.-H. Ulbrich, Vollgraduierte Algebren, Abhand. Math. Sem. Universität Hamburg, 51 (1981), 136-148.

[U2] , Galois erweiterungen von nicht-kommutativen ringen, Comm. Algebra, 10 (1982), 655-672.

[V] M. Van den Bergh, A duality theorem for Hopf algebras, in Methods in Ring Theory, Proceedings of the 1983 NATO ASI in Antwerp, Reidel, 1984.

Received March 4, 1988. The second author was partially supported by NSF Grant DMS 87-00641.

UNIVERSITY OF CALIFORNIA

Los ANGeles, CA 90024-1555

AND

UNIVERSITY OF SOUTHERN CALIFORNIA

LOS ANGELES, CA 90089-1113 


\section{PACIFIC JOURNAL OF MATHEMATICS EDITORS}

\author{
V. S. VARADARAJAN \\ (Managing Editor) \\ University of California \\ Los Angeles, CA 90024 \\ Herbert Clemens \\ University of Utah \\ Salt Lake City, UT 84112 \\ THOMAS ENRIGHT \\ University of California, San Diego \\ La Jolla, CA 92093
}

R. FINN

Stanford University

Stanford, CA 94305

HeRmanN FlaschKa

University of Arizona

Tucson, AZ 85721

VAUGHAN F. R. JONES

University of California

Berkeley, CA 94720

STEVEN KeRCKHOFF

Stanford University

Stanford, CA 94305
RoBION KIRBY

University of California

Berkeley, CA 94720

C. C. MOore

University of California

Berkeley, CA 94720

HAROLD STARK

University of California, San La Jolla, CA 92093

\section{ASSOCIATE EDITORS}
R. ARENS
E. F. BECKENBACH
B. H. NeUmanN
F. WolF
K. YosHIDA (1906-1982)

\section{SUPPORTING INSTITUTIONS}
UNIVERSITY OF ARIZONA
UNIVERSITY OF BRITISH COLUMBIA CALIFORNIA INSTITUTE OF TECHNOLOGY UNIVERSITY OF CALIFORNIA MONTANA STATE UNIVERSITY UNIVERSITY OF NEVADA, RENO NEW MEXICO STATE UNIVERSITY OREGON STATE UNIVERSITY
UNIVERSITY OF OREGON UNIVERSITY OF SOUTHERN CALIFORNIA STANFORD UNIVERSITY UNIVERSITY OF HAWAII UNIVERSITY OF TOKYO UNIVERSITY OF UTAH WASHINGTON STATE UNIVERSITY UNIVERSITY OF WASHINGTON 


\section{Pacific Journal of Mathematics}

\section{Vol. 137, No. $1 \quad$ January, 1989}

V. S. Varadarajan, Henry Abel Dye $\ldots \ldots \ldots \ldots \ldots \ldots \ldots \ldots \ldots \ldots$ iii

Huzihiro Araki, An application of Dye's theorem on projection lattices to orthogonally decomposable isomorphisms $\ldots \ldots \ldots \ldots \ldots \ldots \ldots \ldots \ldots$

Richard Arens, The limit of a sequence of squares in an algebra need not be

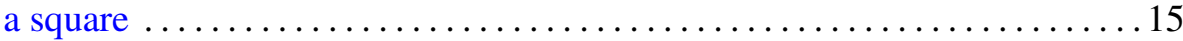

William Arveson, An addition formula for the index of semigroups of

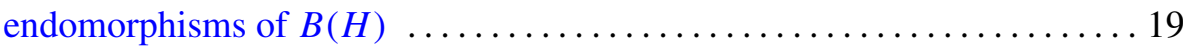

Robert James Blattner and Susan Montgomery, Crossed products and

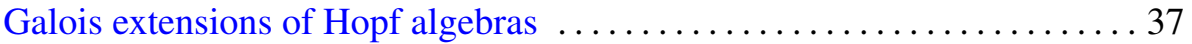

Erik Christensen and Allan M. Sinclair, On the vanishing of $H^{n}\left(\mathscr{A}, \mathscr{A}^{*}\right)$

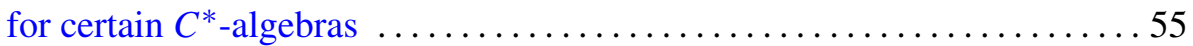

Philip C. Curtis, Jr. and Michael M. Neumann, Nonanalytic functional

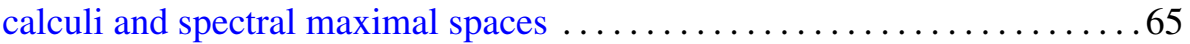

George A. Elliott and David E. Handelman, Addition of $C^{*}$-algebra extensions

Yaakov Friedman and Bernard Russo, Some affine geometric aspects of

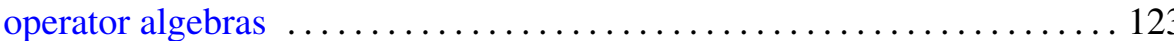

Valentin Ya. Golodets and Sergey D. Sinelshchikov, Regularization of actions of groups and groupoids on measured equivalence relations . . . 145

Irving Kaplansky, CCR-rings 155

Hideki Kosaki, Characterization of crossed product (properly infinite case)

Gert Kjærgaard Pedersen, Three quavers on unitary elements in $C^{*}$-algebras

Sorin Popa, Relative dimension, towers of projections and commuting squares of subfactors

Martin E. Walter, On a new method for defining the norm of

Fourier-Stieltjes algebras 